\title{
Can Routine Offering of Influenza Vaccination in Office-Based Settings Reduce Racial and Ethnic Disparities in Adult Influenza Vaccination?
}

\author{
Jürgen Maurer, $P h D^{1,2}$, Katherine M. Harris, $P h D^{3,2}$, and Lori Uscher-Pines, $P h D^{2}$ \\ ${ }^{1}$ Institute of Health Economics and Management, University of Lausanne, Lausanne, Switzerland; ${ }^{2}$ RAND Corporation, Arlington, VA, USA; \\ ${ }^{3}$ MedStar Health Research Institute, Hyattsville, MD, USA.
}

BACKGROUND: Influenza vaccination remains below the federally targeted levels outlined in Healthy People 2020. Compared to non-Hispanic whites, racial and ethnic minorities are less likely to be vaccinated for influenza, despite being at increased risk for influenzarelated complications and death. Also, vaccinated minorities are more likely to receive influenza vaccinations in office-based settings and less likely to use non-medical vaccination locations compared to non-Hispanic white vaccine users.

OBJECTIVE: To assess the number of "missed opportunities" for influenza vaccination in office-based settings by race and ethnicity and the magnitude of potential vaccine uptake and reductions in racial and ethnic disparities in influenza vaccination if these "missed opportunities" were eliminated.

DESIGN: National cross-sectional Internet survey administered between March 4 and March 14, 2010 in the United States.

PARTICIPANTS: Non-Hispanic black, Hispanic and non-Hispanic white adults living in the United States $(\mathrm{N}=3,418)$.

MAIN MEASURES: We collected data on influenza vaccination, frequency and timing of healthcare visits, and selfreported compliance with a potential provider recommendation for vaccination during the 2009-2010 influenza season. "Missed opportunities" for seasonal influenza vaccination in office-based settings were defined as the number of unvaccinated respondents who reported at least one healthcare visit in the Fall and Winter of 20092010 and indicated their willingness to get vaccinated if a healthcare provider strongly recommended it. "Potential vaccine uptake" was defined as the sum of actual vaccine uptake and "missed opportunities."

KEY RESULTS: The frequency of "missed opportunities" for influenza vaccination in office-based settings was significantly higher among racial and ethnic minorities than non-Hispanic whites. Eliminating these "missed opportunities" could have cut racial and ethnic disparities in influenza vaccination by roughly one half.

Electronic supplementary material The online version of this article (doi:10.1007/s11606-014-2965-z) contains supplementary material, which is available to authorized users.

Received December 8, 2013

Revised March 21, 2014

Accepted July 3, 2014

Published online August 26, 2014
CONCLUSIONS: Improved office-based practices regarding influenza vaccination could significantly impact Healthy People 2020 goals by increasing influenza vaccine uptake and reducing corresponding racial and ethnic disparities.

KEY WORDS: influenza vaccination; racial and ethnic disparities; healthcare provider behavior.

J Gen Intern Med 29(12):1624-30

DOI: $10.1007 / \mathrm{s} 11606-014-2965-\mathrm{z}$

(C) Society of General Internal Medicine 2014

\section{INTRODUCTION}

Influenza is a common, contagious respiratory illness caused by influenza viruses. Individuals with medical conditions such as heart disease, hypertension, cancer, asthma, and diabetes are at increased risk of influenza-related complications and death. ${ }^{1}$ Depending on the severity of annual outbreaks, influenza and related complications, such as pneumonia are responsible for between 3,000 and 50,000 deaths per year in the United States alone. ${ }^{2}$ Because chronic conditions are more prevalent among racial and ethnic minorities, influenza places minorities at greater risk of illness and death. ${ }^{3}$

Despite higher risks, U.S. minority adults are less likely to be vaccinated for influenza than non-Hispanic whites. ${ }^{4}$ While overall adult vaccination rates for influenza appeared to increase following the implementation of the U.S. Centers for Disease Control and Prevention's (CDC) universal recommendation for influenza vaccination in 2010, minority adults continue to be vaccinated at rates that lag behind those of whites by roughly ten percentage points. ${ }^{1,5}$

The literature on reducing disparities in influenza vaccination often focuses on the need to change negative attitudes about vaccination among minorities. ${ }^{6}$ Patient-provider interactions may constitute key intervention points for changing negative attitudes about vaccination and increasing vaccine uptake among minorities. Healthcare professionals are typically the most common and trusted source of health information and often influence patients' decisions of whether or not to be vaccinated for influenza. ${ }^{7-12}$ Emphasizing the prominent 
role of healthcare providers in encouraging influenza vaccination, CDC stresses that "vaccination coverage can be increased by administering vaccine before and during the influenza season to persons during hospitalizations or routine healthcare visits" and recommends that corresponding "vaccination efforts should begin as soon as vaccine is available and continue through the influenza season, which typically extends through April". 1

Demonstrating the predominant role of physician offices for vaccinating minorities, recent research has shown that vaccinated minorities rely relatively more frequently on medical rather than non-medical settings, such as worksite vaccination, or drug or grocery stores for getting influenza vaccinations than vaccinated non-Hispanic whites. ${ }^{13,14}$ Moreover, minority adults appear to have comparable levels of vaccination acceptance compared to non-Hispanic whites when offered influenza vaccination in medical settings, even though they often report negative attitudes toward influenza vaccination. $6,12,15$ Thus, efforts to improve the frequency and effectiveness of offers and recommendations to be vaccinated during medical visits, such as standing order programs or electronic decision support systems, have the potential to substantially increase vaccination rates among minorities and reduce vaccination disparities. ${ }^{16}$

To assess the potential impact of routine vaccination offerings in medical settings on influenza vaccination levels and disparities, we present national estimates of the proportion of non-Hispanic white ("whites"), nonHispanic black ("blacks"), and Hispanic adults who were amenable to seasonal influenza vaccination, but remained unvaccinated-so-called "missed opportunities"-during the 2009-2010 pandemic influenza season. We also estimated potential uptake of seasonal influenza vaccination by race and ethnicity if "missed opportunities" for seasonal influenza vaccination were eliminated. ${ }^{15,17}$ Since pandemic vaccination was more frequently administered outside officebased settings for logistical reasons, our analysis only focuses on "missed opportunities" for seasonal influenza vaccination, which was widely available in medical settings.

\section{METHODS}

Between March 4 and March 24, 2010, we fielded a short influenza vaccination survey to adult members of a nationally representative online panel of US households managed by Knowledge Networks, Inc., Menlo Park, CA (KN). ${ }^{18}$ KN uses a probability-based sampling scheme that combines randomdigit-dialing and address-based sampling to recruit panelists in order to cover both cell-phone-only and "offline" households. ${ }^{18}$ To ensure a sufficient representation of older adults and ethnic diversity, we oversampled adults age 65 and older as well as black and Hispanic participants. Seventy-four percent of sampled panelists responded to our survey, yielding an overall sample of 4,040 respondents. We excluded observations with missing values on our main outcomes of interest (139 observations) from our analysis, as well as observations who did not self-identify as non-Hispanic black, Hispanic or non-Hispanic white (483 observations), for a final analytical sample of 3,418 U.S. adults. Table 1 presents both unweighted and weighted characteristics of our final sample. The unweighted characteristics reflect our sample stratification, while the weighted characteristics are computed using post-stratification weights based on data from the U.S. Current Population Survey to adjust for known sampling probabilities, sample stratification, non-response to panel recruitment and panel attrition, as well as to ensure that our final data match selected socio-demographic characteristics of the U.S. adult population. ${ }^{19}$

The survey started with an introduction about influenza, highlighting the existence of two distinct vaccinations (so-called "seasonal" and "pandemic"/"H1N1/Swine flu") during the 2009-2010 influenza season. The survey introduction further disclosed the study sponsor (GlaxoSmithKline) and noted that the response to the survey, or any individual question on the survey, was completely voluntary. The Institutional Review Board of the RAND Corporation approved the questionnaire and study design.

Table 1. Overview of Selected Sample Characteristics, U.S. Adults, March 2010, N=3,418

\begin{tabular}{|c|c|c|}
\hline \multirow[t]{2}{*}{ Characteristic } & \multicolumn{2}{|c|}{ Sample frequencies } \\
\hline & Unweighted n & $\begin{array}{l}\text { Weighted \% } \\
(95 \% \text { CI) }\end{array}$ \\
\hline \multicolumn{3}{|l|}{ Age } \\
\hline $18-29$ & 254 & 21.0 \\
\hline $30-44$ & 450 & 28.4 \\
\hline $45-59$ & 1,174 & 25.8 \\
\hline $60+$ & 1,540 & 24.8 \\
\hline \multicolumn{3}{|l|}{ Race/Ethnicity } \\
\hline White & 1,760 & 74.0 \\
\hline Black & 1,089 & 11.5 \\
\hline Hispanic & 569 & 14.5 \\
\hline \multicolumn{3}{|l|}{ Sex } \\
\hline Male & 1,587 & 48.7 \\
\hline Female & 1,831 & 51.3 \\
\hline \multicolumn{3}{|l|}{ Education } \\
\hline Less than high school & 320 & 12.7 \\
\hline High school & 886 & 31.5 \\
\hline Some college & 1,131 & 28.8 \\
\hline College degree or higher & 1,081 & 27.0 \\
\hline \multicolumn{3}{|l|}{ Work status } \\
\hline Employed & 1,369 & 49.1 \\
\hline Self-employed & 252 & 7.0 \\
\hline Temporary leave & 40 & 2.0 \\
\hline Unemployed & 205 & 8.1 \\
\hline Retired & 1,051 & 16.7 \\
\hline Disabled & 330 & 9.5 \\
\hline Other non-working & 171 & 7.7 \\
\hline \multicolumn{3}{|c|}{ Internet access (before panel recruitment) } \\
\hline Yes & 2,497 & 65.7 \\
\hline No & 921 & 34.3 \\
\hline
\end{tabular}

${ }^{a}$ The poststratification weights were computed using data from the U.S. Current Population Survey and were adjusted for known sampling probabilities; sample stratification; and non-response to panel recruitment and panel attrition. ${ }^{19}$ 
Uptake of seasonal influenza vaccine was assessed based on the survey item "Did you get a seasonal flu vaccine this past flu season (August 2009 to March 2010)?” Respondents were also asked: "How many times did you go to a doctor's office or clinic to get care for yourself between September 2009 and March 2010," as well as the exact months during which these visits occurred. Finally, unvaccinated persons who did not report receiving a vaccination recommendation for seasonal influenza from a healthcare provider were asked: "Would you get a seasonal flu vaccine if a doctor or health care provider strongly recommended it to you?" in order to assess their willingness to get vaccinated under improved patient-provider communication.

We defined "missed opportunities" for seasonal vaccination as the number of respondents who were (a) unvaccinated for seasonal influenza; (b) reported at least one visit to a healthcare provider between September 2009 and December 2009, January 2010 or February 2010, respectively; and (c) indicated their willingness to get vaccinated for seasonal influenza if a healthcare provider strongly recommended it to them. Defining "missed opportunities" for seasonal influenza vaccination based only on healthcare provider visits that occurred between September and December 2009 represents a fairly conservative approach to measurement, as it only considers the most frequent vaccination months during which influenza vaccine is typically widely available in officebased settings. On the other hand, expanding the definition of missed opportunities to also include January and February of 2010 as potential vaccination months is more aligned with the U.S. Centers for Disease Control and Prevention (CDC)'s vaccination practice recommendations and provides a useful robustness check to differences in measurement. Finally, we defined "potential vaccine uptake" as the sum of actual vaccine uptake and missed opportunities as defined above.

We used proportion estimation to obtain unadjusted outcomes by race and ethnicity, which ensured that our estimated counterfactual of "potential vaccine uptake" can be directly compared to published statistics on racial and ethnic disparities in adult influenza vaccination and corresponding policy targets. ${ }^{20,21}$ The statistical significance of differences in seasonal vaccine uptake, healthcare provider contact during the fall and winter, missed opportunities for vaccination and potential vaccine uptake across racial and ethnic groups was assessed based on Wald- and $\chi^{2}$ - tests. We also estimated average partial effects of multivariable probit models for the above outcomes, to provide a more complete analysis of other potential sociodemographic predictors of seasonal vaccine uptake, healthcare provider contact during the fall and winter, missed opportunities for vaccination and potential vaccine uptake, and to assess whether or bivariate results regarding differences across racial and ethnic groups are also obtained once other sociodemographic characteristics are taken into account. Due to some item non-response in these socioeconomic predictors, we lost another ten observations for those additional multivariable analyses. All estimates were generated using STATA 12.1 SE software (StataCorp, College Station, TX). All reported estimates were weighted using post-stratification weights derived from the Current Population Survey that adjust for known selection probabilities; sample stratification; non-response to panel recruitment; and panel attrition. ${ }^{19}$

\section{RESULTS}

Table 2 shows that seasonal vaccine uptake during the 20092010 influenza season varied significantly across racial and ethnic groups $(p=0.001)$. Compared to non-Hispanic whites, seasonal vaccine uptake was significantly lower among blacks (42.6\% vs. $32.6 \%, \mathrm{p}=0.005)$ and Hispanics (42.6\% vs. $30.1 \%, p=0.002)$. At the same time, the fraction of adults with at least one contact with a healthcare provider during fall and winter did not differ significantly across the three racial and ethnic groups, irrespective of the exact time period considered.

By contrast, "missed opportunities" for vaccination were significantly higher among minorities compared to nonHispanic whites, with $\mathrm{p}$ values for tests of equal proportions across the three racial and ethnic groups ranging from $\mathrm{p}=0.019$ to $\mathrm{p}=0.040$ for the different time periods used for defining "missed opportunities." Despite some expected loss in statistical power, direct two-group comparisons between whites and blacks and whites and Hispanics still revealed statistically significant differences at the $10 \%$ significance level.

Irrespective of the considered time period, potential uptake of seasonal vaccine achievable by eliminating "missed opportunities" did not differ statistically significantly across the three racial and ethnic groups at any conventional level of significance, which indicates that the higher incidence of "missed opportunities" for vaccination among minorities could partially close the previously documented racial and ethnic disparities in influenza vaccination, yielding more comparable rates of "potential vaccine uptake."

Table 3 reports average partial effects from multivariable models for seasonal vaccine uptake, healthcare provider contact during the fall and winter, missed opportunities for vaccination and potential vaccine uptake on race and ethnicity and other sociodemographic controls. To conserve space, we only reported estimates measuring healthcare provider contacts and corresponding missed opportunities between September 2009 and January 2010. The Online Appendix to this paper presents more detailed estimation results, including those from similar models, using our alternative time horizons through December 2009 and February 2010 for defining fall and winter provider contact and missed opportunities, respectively.

The first column of Table 3 shows that-holding other characteristics fixed - minorities still displayed roughly five to six percentage points lower levels of seasonal vaccine 
Table 2. Influenza Vaccine Uptake, Any Healthcare Provider Contact, Missed Opportunities for Vaccination, and Potential Vaccination Rates Among U.S. Adults by Race and Ethnicity, N=3,418, March 2010

\begin{tabular}{|c|c|c|c|c|c|c|}
\hline \multirow{3}{*}{$\begin{array}{l}\text { Number of observations n } \\
\text { Characteristic }\end{array}$} & \multirow{3}{*}{$\begin{array}{l}\text { White \% (95\% CI) } \\
1,760 \\
\text { Adults }\end{array}$} & \multirow{2}{*}{\multicolumn{2}{|c|}{$\begin{array}{l}\text { Black \% }(95 \% \text { CI }) \\
1,089\end{array}$}} & \multirow{2}{*}{\multicolumn{2}{|c|}{$\begin{array}{l}\text { Hispanic \% (95 \% CI) } \\
569\end{array}$}} & \multirow[b]{3}{*}{ p value } \\
\hline & & & & & & \\
\hline & & Adults & p value ${ }^{a}$ & Adults & p value e $^{a}$ & \\
\hline Vaccinated for seasonal influenza & $42.6(39.2-46.0)$ & $32.6(26.5-38.7)$ & 0.005 & $30.1(22.8-37.4)$ & 0.002 & 0.001 \\
\hline \multicolumn{7}{|c|}{ Any healthcare provider contact during fall and winter ${ }^{\mathrm{c}}{ }^{\mathrm{V}}$} \\
\hline September 2009 to December 2009 & $56.1(52.6-59.6)$ & $60.2(53.8-66.7)$ & 0.273 & $59.5(51.2-67.9)$ & 0.457 & 0.498 \\
\hline September 2009 to January 2010 & $62.0(58.5-65.5)$ & $66.5(60.2-72.9)$ & 0.222 & $64.0(55.9-72.1)$ & 0.654 & 0.543 \\
\hline September 2009 to February 2010 & $64.9(61.5-68.4)$ & $71.8(66.0-77.7)$ & 0.047 & $67.4(59.5-75.4)$ & 0.584 & 0.257 \\
\hline \multicolumn{7}{|c|}{ Missed opportunities during fall and winter ${ }^{\mathrm{d}}$} \\
\hline September 2009 to December 2009 & $10.6(8.3-13.0)$ & $14.7(10.9-18.4)$ & 0.073 & $17.3(10.3-24.3)$ & 0.077 & 0.040 \\
\hline September 2009 to January 2010 & $12.2(9.7-14.7)$ & $16.3(12.2-20.4)$ & 0.096 & $20.2(12.5-27.8)$ & 0.052 & 0.026 \\
\hline September 2009 to February 2010 & $12.7(10.1-15.2)$ & $17.5(13.2-21.7)$ & 0.059 & $21.0(13.3-28.7)$ & 0.045 & 0.019 \\
\hline \multicolumn{7}{|l|}{ Potential vaccine uptake $\mathrm{e}^{\mathrm{e}}$} \\
\hline September 2009 to December 2009 & $53.3(49.7-56.8)$ & $47.3(40.9-53.7)$ & 0.108 & $47.4(39.0-55.7)$ & 0.203 & 0.184 \\
\hline September 2009 to January 2010 & $54.8(51.3-58.3)$ & $48.9(42.5-55.3)$ & 0.112 & $50.3(41.9-58.6)$ & 0.323 & 0.262 \\
\hline September 2009 to February 2010 & $55.3(51.8-58.8)$ & $50.1(43.6-56.5)$ & 0.163 & $51.1(42.7-59.4)$ & 0.360 & 0.336 \\
\hline
\end{tabular}

Notes: ${ }^{a} p$ values from a Wald-test of statistical differences of subpopulation rates between whites and African Americans and whites and Hispanics, respectively

${ }^{b} \chi^{2}$-test for significant differences across all three racial and ethnic groups

${ }^{c}$ Healthcare provider contact during fall and winter: at least one visit to a healthcare provider from September 2009 to December 2009 , January 2010 or February 2010, respectively

${ }^{d}$ Missed opportunities during fall and winter: unvaccinated for seasonal with at least one visit to a healthcare provider during the fall and winter from September 2009 to December 2009, January 2010 or February 2010, respectively) and self-reported willingness to be vaccinated for seasonal influenza with a strong provider recommendation

${ }^{e}$ Potential vaccine uptake: actual vaccine uptake + missed opportunities

uptake, although these estimates were only statistically significant for blacks and only at the $10 \%$ significance level. Moreover, persons with higher household incomes and health insurance, as well as older persons, persons with chronic conditions, healthcare workers and persons with regular contact to high-risk individuals, were significantly more like to be vaccinated.

Table 3. Average Partial Effects (APE) of Multivariable Probit Models for Influenza Vaccine Uptake, Any Healthcare Provider Contact, Missed Opportunities for Vaccination, and Potential Vaccine Uptake Among U.S. adults by Race and Ethnicity, N=3,408, March 2010

\begin{tabular}{|c|c|c|c|c|c|c|c|c|}
\hline \multirow[t]{2}{*}{ Characteristic } & \multicolumn{2}{|c|}{$\begin{array}{l}\text { Seasonal vaccine } \\
\text { uptake (through } \\
\text { March) }\end{array}$} & \multicolumn{2}{|c|}{$\begin{array}{l}\text { Any healthcare } \\
\text { provider contact }^{\mathrm{a}} \\
\text { during fall and } \\
\text { winter (September } \\
\text { through January) }\end{array}$} & \multicolumn{2}{|c|}{$\begin{array}{l}\text { Missed } \\
\text { opportunities } \\
\text { for vaccination } \\
\text { during fall and } \\
\text { winter (September } \\
\text { through January) }\end{array}$} & \multicolumn{2}{|c|}{$\begin{array}{l}\text { Potential vaccine } \\
\text { uptake during } \\
\text { fall and winter }\end{array}$} \\
\hline & $\begin{array}{l}\text { Averag } \\
\text { Effect }^{d}\end{array}$ & & $\begin{array}{l}\text { Averag } \\
\text { Effect }^{d}\end{array}$ & & $\begin{array}{l}\text { Average } \\
\text { Effect }^{\text {d }}\end{array}$ & & $\begin{array}{l}\text { Averag } \\
\text { Effect }^{d}\end{array}$ & \\
\hline Black & -0.063 & $\dagger$ & 0.081 & $*$ & 0.037 & & -0.025 & \\
\hline Hispanic & -0.059 & & 0.087 & $*$ & 0.072 & $*$ & 0.021 & \\
\hline Age $50-64$ & 0.088 & $* *$ & 0.068 & $*$ & -0.04 & & 0.042 & \\
\hline Age 65 and older & 0.246 & $* * *$ & 0.133 & $* *$ & -0.081 & $*$ & 0.185 & $* * *$ \\
\hline Female & 0.015 & & 0.078 & $* *$ & 0.028 & & 0.043 & \\
\hline Married/partnered & 0.013 & & -0.017 & & -0.027 & & -0.009 & \\
\hline Any children & 0.002 & & -0.022 & & -0.02 & & -0.025 & \\
\hline Rural area & -0.03 & & -0.006 & & -0.012 & & -0.047 & \\
\hline Some college & 0.017 & & -0.073 & $*$ & -0.037 & & -0.018 & \\
\hline Bachelor or higher & 0.018 & & -0.069 & $*$ & -0.012 & & 0.009 & \\
\hline Employed & -0.048 & & -0.050 & & 0.011 & & -0.039 & \\
\hline Income $\$ 50-100 \mathrm{~K}$ & 0.073 & $*$ & -0.009 & & -0.024 & & 0.047 & \\
\hline Income $\$ 100 \mathrm{~K}+$ & 0.092 & $*$ & 0.083 & $*$ & 0.009 & & 0.094 & \\
\hline Health insurance & 0.098 & $*$ & 0.229 & $* * *$ & 0.07 & $*$ & 0.155 & $* * *$ \\
\hline Chronic condition & 0.159 & $* * *$ & 0.223 & $* * *$ & 0.028 & & 0.192 & $* * *$ \\
\hline Healthcare worker & 0.186 & $* * *$ & 0.029 & & -0.048 & & 0.155 & $* * *$ \\
\hline High-risk contact & 0.071 & $* *$ & 0.071 & $*$ & 0.003 & & 0.073 & $*$ \\
\hline
\end{tabular}

Average Partial Effects represent average changes in probabilities associated with a unit change in any given variable and are therefore bounded between -1 and 1 Notes: ${ }^{a}$ Healthcare provider contact during fall and winter: At least one visit to a healthcare provider from September 2009 to December 2009 , January 2010 or February 2010, respectively

${ }^{b}$ Missed opportunities during fall and winter: Unvaccinated for seasonal with at least one visit to a healthcare provider during the fall and winter from September 2009 to December 2009, January 2010 or February 2010, respectively), and self-reported willingness to be vaccinated for seasonal influenza with a strong provider recommendation

${ }^{c}$ Potential vaccine uptake: Actual vaccine uptake + missed opportunities

${ }^{d}$ Indicators for statistical significance: $+: p<0.1 ; *: p<0.05 ; * *: p<0.01 ; * * *: p<0.001$ 
The second column of Table 3 presents estimates of the occurrence of any healthcare provider contact during the fall and winter as a necessary condition for a missed opportunity for provider-based vaccinations. These estimates show that minorities had a statistically significantly higher average probability of at least one healthcare provider contact between September 2009 and January 2010 than non-Hispanic whites, once we adjusted for other respondent characteristics. The adjusted average probability of any healthcare provider contact during the fall and winter was also statistically significantly higher among older persons, women, persons with relatively low education, higher incomes and health insurance, as well as persons with chronic conditions and regular contacts of persons at high risk for influenza-related complications.

Missed opportunities for vaccination, on the other hand, showed relatively few statistically significant partial associations with respondents' sociodemographic characteristics (Table 3, column 3). While missed opportunities for vaccination still appeared to be more concentrated among minorities after adjusting for other sociodemographic characteristics, the estimated partial effect of being in a minority group was only statistically significant for Hispanics. Adjusted levels of missed opportunities were also statistically significantly higher among persons with health insurance and statistically significantly lower among older respondents. None of the other sociodemographic controls entered the model for missed opportunities statistically significantly.

Lastly, column 4 of Table 3 explores potential sociodemographic predictors of potential vaccine uptake defined as either being vaccinated or having had a missed opportunity for vaccination. Similar to our previous bivariate analyses, potential vaccine uptake did not display any statistically significant adjusted differences by race and ethnicity. At the same time, eliminating all missed opportunities for vaccination would still have resulted in statistically significantly higher adjusted vaccination rates among older persons, persons with health insurance, persons with chronic conditions, healthcare workers and regular contacts of persons at elevated risk for influenza-related complications.

\section{DISCUSSION}

CDC recommends that healthcare providers offer influenza vaccination to all adult patients during influenza season. ${ }^{1}$ Our findings suggest that routine offering of influenza vaccine to amenable patients in office-based settings has the potential to increase the uptake of influenza vaccine among all adults and cut racial and ethnic disparities by roughly one half. These reductions in racial and ethnic disparities do not solely stem from other personal characteristics that are correlated with race and ethnicity, as multivariable probit models that control for other socio-demographic respondent characteristics still indicated a positive partial association between missed opportunities for office-based vaccination and minority status. By contrast, other socioeconomic disparities in influenza vaccination - notably those related to health insurance status - would remain, even if all missed opportunities for vaccination in office-based settings could be successfully eliminated.

Our findings reinforce calls for a more systematic offering of influenza vaccine to reduce corresponding vaccination disparities across racial and ethnic groups. ${ }^{22}$ For example, published data from the 1995/1996 Medicare Current Beneficiary Surveys and the 2007 National immunization Survey show that older minority adults are less likely to be vaccine seekers than older non-Hispanic whites. ${ }^{15,22}$ Responding to this challenge, routine offering of influenza vaccine in officebased settings can reduce racial and ethnic disparities in influenza vaccination by moving the responsibility for initiating vaccination-related patient-provider interactions from patients to providers. Proactive, provider-based vaccination counseling during routine office visits seems especially important for reducing such disparities, since non-vaccineseeking minorities are also unlikely to get vaccinated in alternative vaccination settings such as grocery stores, pharmacies or retail settings that crucially rely on vaccineseeking behaviors by consumers. More limited active vaccine-seeking behavior among minorities is also consistent with the observation that racial and ethnic disparities in influenza vaccination tend to be larger when influenza vaccine is in short supply. ${ }^{23,24}$ As a result, more systematic offering of influenza vaccine in office-based settings may lead to reductions in racial and ethnic disparities in influenza vaccination that stem from demand-side factors, similar to the large progress in reducing vaccination disparities by race and ethnicity for children following the introduction of the Vaccines for Children Program in the mid-1990s. ${ }^{25}$

Our study suffers from several shortcomings. First, our analysis used self-reported data and was in part based on a question referring to a hypothetical scenario, whose accuracy is not known. Second, our data were collected using the Internet as survey mode, which may result in biases if participation in the survey is systematically related to influenza vaccination status or vaccination-related attitudes. While $\mathrm{KN}$ makes every effort to ensure representativeness of their Internet panel by employing address-based and phone-based sampling rather than following an opt-in approach with unknown sampling frame, our data may nonetheless not be fully representative of the entire adult population, especially for racial and ethnic minorities. Despite the fact that our current estimates of seasonal vaccine uptake are close to those from other sources ${ }^{20}$ and that data from our and comparable Internet surveys have been widely published in the literature, ${ }^{14,17,21,26}$ it would therefore nonetheless be useful to replicate our analysis on other nationally representative data to assess the robustness of our findings with respect to sampling method and survey mode. Third, our data were collected during the 20092010 pandemic influenza season, i.e., during a time of increased attention to influenza and influenza vaccination, 
which may challenge the external validity of our findings. Fourth, while routine offering of influenza vaccine can result in significant progress toward various health policy goals of Healthy People 2020 and is thus interesting in its own right, ${ }^{27}$ our study does not provide any insights on the relative costs and benefits of routine offering of influenza vaccine as compared to, say, other potentially underused interventions during medical encounters, such as counseling for smoking cessation, weight loss or reproductive health. Beyond the scope of this paper, a comprehensive cost-benefit analysis of routine offering of influenza vaccine in medical settings would certainly be desirable. Despite these limitations, our study provides unique insights on how routine offering of influenza vaccine in officebased settings could affect racial and ethnic disparities in vaccine uptake, thanks to its detailed information on vaccination status, healthcare use and the exact timing of these healthcare visits and respondents' self-reported willingness to get vaccinated in case of a vaccination offer, which are typically not available in health surveys.

Given its large potential to increase vaccine uptake and narrow corresponding racial and ethnic disparities, one may wonder about potential reasons why routine offering of influenza vaccine to amenable patients in office-based settings remains underused. First, competing time demands may result in underuse of effective and desirable interventions for improving care quality in general, and potential lack of priority may make this issue particularly severe for the routine offering of influenza vaccine. ${ }^{24,28}$ This issue may be further exacerbated in the context of vaccinating minority patients if physicians fear higher levels of vaccine resistance in these patient groups, despite conflicting empirical evidence on this issue to date. ${ }^{12,15}$ Second, better reimbursement of physicians for vaccine purchases, vaccination counseling and administration may also improve vaccination-related behavior in office-based settings. ${ }^{24}$ In addition, potential lack of effective reminder systems, the previous need to target vaccination to specific recommendation groups defined based on complex interactions between age and other health risk factors, and lack of corresponding information technology (IT) infrastructure may further exacerbate this issue. The new universal vaccination recommendation of 2010 and increased investments in health IT may thereby facilitate the future routine offering of influenza vaccine considerably. ${ }^{29,30}$ With regard to racial and ethnic disparities, higher levels of care fragmentation, less qualified physicians treating minorities, and more challenging practice settings for delivering primary care to minorities may also play an important role in explaining higher levels of missed opportunities among blacks and Hispanics. ${ }^{31-33}$

Further increasing the annual uptake of influenza vaccine as outlined in the Federal Healthy People 2020 Objectives will likely require the development and implementation of tailored communication strategies aimed at increasing minorities' confidence in the safety and efficacy of vaccination. What is compelling about the potential reductions in vaccination disparities presented here is that they appear relatively easily achievable now through improved office-based practices, which are especially important for vaccinating minorities. ${ }^{13,14}$

\begin{abstract}
Acknowledgements: The National Institute of Allergy and Infectious Diseases (NIAID) (grant R03AI095084) supported this study. The survey data used in this study were collected under contract with GlaxoSmithKline (GSK). GSK had no role in the design, management, analysis, or interpretation of these data, and had no role in the preparation, review, or approval of the article. The opinions expressed here are solely those of the authors and do not represent those of the University of Lausanne, the RAND Corporation, MedStar, GSK, or NIAID.
\end{abstract}

Conflict of Interest: The authors declare that they do not have a conflict of interest.

Corresponding Author: Jürgen Maurer, $\mathrm{PhD}$; Jürgen Maurer, $\mathrm{PhD}$; IEMS Lausanne, Internef, Dorigny 101 Lausanne, Switzerland (e-mail: Jurgen.maurer@unil.ch).

\section{REFERENCES}

1. Fiore T, Uyeki T, Broder $\mathbf{K}$, et al. Prevention and control of seasonal influenza with vaccines: recommendations of the Advisory Committee on Immunization Practices. 2010. MMWR Recomm Rep. 2010;59 (RR-8): 1-62.

2. Thompson MG, Shay DK, Zhou H, et al. Estimates of deaths associated with seasonal influenza-United States, 1976-2007. MMWR. 2010;59(33): 1057-62.

3. Hutchins SS, Fiscella $\mathbf{K}$, Levine $\mathbf{R}$, et al. Protection of racial/ethnic minority populations during an influenza pandemic. Am J Public Health. 2009;99(suppl 2):S261-70.

4. U.S. Centers for Disease Control and Prevention. Self-reported influenza vaccination coverage trends 1989-2008 among adults by age group, risk group, race/ethnicity, health-care worker status, and pregnancy status, United States, National Health Interview Survey (NHIS). Available online at: http://www.cdc.gov/flu/pdf/professionals/nhis89_08fluvaxtrendtab.pdf (accessed 4 July 2014).

5. U.S. Centers for Disease Control and Prevention. March Flu Vaccination Coverage United States, 2011-12 Influenza Season. Available online at: http://www.cdc.gov/flu/pdf/fluvaxview/national-flu-survey-mar2012.pdf (accessed 4 July 2014).

6. Lindley MC, Wortley PM, Winston CA, Bardenheier BH. The role of attitudes in understanding disparities in adult influenza vaccination. Am J Prev Med. 2006;31(4):281-5.

7. Hesse BW, Nelson DE, Kreps GL, et al. Trust and sources of health information. The impact of the internet and its implications for health care providers: findings from the first health information national trends survey. Arch Intern Med. 2005;165(22):2618-24.

8. Maurer $\mathbf{J}$, Harris KM. Contact and communication with healthcare providers regarding influenza vaccination during the 2009-2010 H1N1 pandemic. Prev Med. 2011;52(6):459-64.

9. Maurer J. Who has a clue to preventing the flu? Unravelling supply and demand effects on the take-up of influenza vaccinations. J Health Econ. 2009;40(3):704-17.

10. Schmitz H, Wübker A. What determines influenza vaccination take-up of elderly Europeans? Health Econ. 2011;20(11):1281-97.

11. Chi RC, Neuzil KM. The association of sociodemographic factors and patient attitudes on influenza vaccination rates in older persons. Am J Med Sci. 2004;327(3): 113-7.

12. Schwartz KL, Neale AV, Northrup J, et al. Racial similarities in response to standardized offer of influenza vaccination: a metronet study. J Gen Intern Med. 2006;21(4):346-51.

13. U.S. Centers for Disease Control and Prevention. Place of Influenza Vaccination Among Adults - United States, 2010-11 Influenza Season. MMWR 2011;60(23):781-785. 
14. Uscher-Pines L, Maurer J, Harris KM. Racial and ethnic disparities in uptake and location of vaccination for 2009-H1N1 and seasonal influenza. Am J Public Health. 2011;101(7):1252-5.

15. Hebert PL, Frick KD, Kane RL, et al. The causes of racial and ethnic differences in influenza vaccination rates among elderly Medicare beneficiaries. Health Serv Res. 2005;40:517-37.

16. U.S. Centers for Disease Control and Prevention. Increasing adult vaccination rates: What works: Strategies list. Available online at: http://www2a.cdc.gov/ vaccines/ed/whatworks/strategies_list.asp (accessed 4 July 2014)

17. Maurer J, Harris KM, Lurie N. Reducing missed opportunities to vaccinate adults against influenza: what is realistic? Arch Intern Med. 2009;169(17):1633-4.

18. GfK. KnowledgePanel Available online at: http://www.gfk.com/Documents/ GfK-KnowledgePanel.pdf (accessed 4 July 2014).

19. Knowledge Networks. Overview of KnowledgePanel statistical weighting protocol. 11 Nov 2009. Available at: www.knowledgenetworks.com/ganp/ docs/KN-Weighting-Synopsis.pdf (accessed 4 July 2014).

20. U.S. Centers for Disease Control and Prevention. CDC health disparities and inequalities report-United States, 2011. MMWR. 2011;60(Suppl):1-113.

21. Harris KM, Maurer J, Kellermann A. Flu vaccine: safe, effective, and mistrusted. N Engl J Med. 2010;363(23):2183-5.

22. Groom HC, Zhang F, Fisher AK, Wortley PM. Differences in adult influenza vaccine-seeking behavior: the roles of race and attitudes. J Public Health Manag Pract. 2014;20(2):246-50.

23. Yoo BK, Kasajima M, Phelps CE, Fiscella K, Bennett NM, Szilagyi PG. Influenza vaccine supply and racial/ethnic disparities in vaccination among the elderly. Am J Prev Med. 2011;40(1):1-10.

24. Yoo BK. How to improve influenza vaccination rates in the U.S. J Prev Med. Public Health. 2011;44(4):141-8.
25. Walker AT, Smith PJ, Kolasa M. Reduction of racial/ethnic disaprities in vaccination coverage, 1995-2011. MMWR. 2014;63(1):7-12.

26. Knowledge Networks. GfK bibliography: Articles and presentations based on GfK's collected panel data, analysis or methodology. Available at: http://www.knowledgenetworks.com/ganp/docs/KN-Bibliography.pdf (accessed 4 July 2014)

27. U.S. Department of Health and Human Services. Healthy People 2020. 2020 Topics \& Objectives. Immunization and Infectious Diseases. Available online at: http://www.healthypeople.gov/2020/topicsobjectives2020/ overview.aspx?topicId=23 (accessed 4 July 2014).

28. Maurer J, Harris KM. Issuance of patient reminders for influenza vaccination by US-based primary care physicians during the first year of universal influenza vaccination recommendations. Am J Public Health. 2014;104(6):e60-2.

29. U.S. Centers for Disease Control and Prevention. Prevention and control of influenza with vaccines: recommendations of the Advisory Committee on Immunization Practices (ACIP), 2010. MMWR. 2010;59(No. RR-8): 1-62.

30. Buntin MB, Burke MF, Hoaglin MC, Blumenthal D. The benefits of health information technology: a review of the recent literature shows predominantly positive results. Health Aff. 2011;30(3):464-71.

31. Oster A, Bindman AB. Emergency department visits for ambulatory care sensitive conditions: insights into preventable hospitalizations. Med Care. 2003;41(2):198-207.

32. Bach PB, Pham HH, Schrag $\mathbf{D}$, et al. Primary care physicians who treat blacks and white. N Engl J Med. 2004;351(6):575-84.

33. Escarce JJ. Racial and ethnic disparities in access to and quality of health care. Robert Wood Johnson Foundation, Research Synthesis Report No. 12 2007:1-32. 